\title{
Dos Fragmentos à Complexidade: Principios para a Construção da Estratégia no Século XXI
}

\author{
From the Fragments to the Complexity: Principles to Build Strategy in the XXI \\ Century
}

\author{
Leonardo Augusto Amaral Terra ${ }^{\mathrm{I}}$ \\ João Luiz Passador ${ }^{2}$
}

\section{Resumo}

Este esforço de pesquisa visa apontar orientações epistemológicas sobre como as investigações na área de gestão devem ser tratadas e as consequências metodológicas dessa mudança de percepção, visando possibilitar o surgimento de um espaço teórico reflexivo, capaz de responder à complexidade dos cenários contemporâneos. Para tanto, foi desenvolvida uma pesquisa teórica, cujo resultado apontou que, para romper com a lógica fragmentadora que permeia a própria estrutura de apreensão do conhecimento na área de gestão, é preciso que as abordagens multimétodos se tornem parte do cotidiano das pesquisas na área da estratégia, estruturadas para abarcar as etapas teóricas, empíricas, indutivas e dedutivas de forma a permitir uma completa compreensão dos fenômenos relacionados ao processo estratégico.

Palavras-chave: Complexidade; Teoria dos Sistemas; Estratégia; Método de Pesquisa; Epistemologia.

\begin{abstract}
The present research aims at pointing out epistemological orientations about how the investigations in the area of management should be addressed, and the methodological consequences of this change of perception, aiming to enable the emergence of a reflective theoretical space capable of responding to the complexity of contemporary scenarios. In order to do so, a theoretical research was developed, and its results pointed out that, in order to break with the fragmentary logic that pervades the very structure of knowledge understanding in the area of management, it is necessary that the multi-method approaches become part of researches in a daily basis in the area of strategy, structured to encompass the theoretical, empirical, inductive and deductive stages in order to allow a complete understanding of the phenomena related to the strategic process.
\end{abstract}

Keywords: Complexity; Systems Theory; Strategy; Research method; Epistemology.

\footnotetext{
1 prof@leoterra.com.br, Brasil. Professor da Universidade de São Paulo - USP. Doutor em Administração de Organizações pela Universidade de São Paulo - USP. Av. Bandeirantes, 3900, Vila Monte Alegre, CEP: 14040-905 - Ribeirão Preto, SP Brasil.

2 jlpassador@usp.br, Brasil. Professor da Universidade de São Paulo - USP. Doutor em Administração de Empresas pela Fundação Getúlio Vargas - FGV. Av. Bandeirantes, 3900, Vila Monte Alegre, CEP: 14040-905 - Ribeirão Preto, SP - Brasil.
}

Recebido em 19.11.2017

Aprovado em 02.04.2018

\section{Revista Administração em Diálogo}

ISSN 2178-0080

PUC-SP 


\section{Introdução}

As afirmações de que o mundo contemporâneo se encontra cada vez mais dinâmico e competitivo têm se tornado corriqueiras no ambiente de gestão, especialmente quando se referem às variáveis diretamente ligadas ao problema estratégico. Os desafios impostos por essa realidade podem ser observados no âmbito das dificuldades que as organizações têm enfrentado ao se deparar com cenários de grande inovação, com as sucessivas crises econômicas pelas quais o mundo tem passado e até mesmo pela complexa dinâmica socioeconômica que tem levado o ser humano a lidar com toda uma gama de problemas ambientais (Senge, Scharmer, Jaworski, \& Flowers, 2008; Taylor \& Tainter, 20I6; Terra, \& Passador, 20I6).

Para Herepath (20I4) e Jarzabkowski e Paul Spee (2009), essa problemática se deve ao fato de que as ligações entre os fenômenos sociais e as ações executivas permanecem subdesenvolvidas, dificultando as ações dos gestores. Farmer e Foley (2009) ainda ressaltam que tais deficiências têm levado os modelos clássicos de gestão a enfrentarem dificuldades ao serem utilizados para criar políticas públicas e organizacionais, pois se baseiam em pressupostos pautados no equilíbrio dinâmico e, por isso, são incompatíveis com essa realidade de constante transformação.

Essa percepção possui uma inquietante analogia com as descrições feitas por Drucker (I994) ao analisar o cenário conturbado da década de I980. Transportando as conclusões do autor para os dias de hoje, pode-se dizer que os velhos mapas e bússolas da realidade, continuam insuficientes para orientar as decisões dos gestores. Para Bertero, Caldas e Wood (I999), a fixação em casos isolados é a principal responsável por essa deficiência. Na busca por soluções específicas, os modelos contemporâneos acabaram se concentrando nas questões diretamente relacionadas com o problema que se pretendia verificar, o que os levaram a ser construídos de forma contornar as mudanças ambientais e não de proporcionar uma ampla compreensão destas (Hurtado, 2006; Mintzberg, Lampel, Quinn, \& Ghoshal, 2003).

$\mathrm{Na}$ área da estratégia, autores como Zey-Ferrell (I98I), Prahalad e Hamel (I994), Drucker (1994), Mintzberg, Ahlstrand e Lampel (1998) Bertero et al. (I999), Bauer (1999), Faria (2005), Hurtado (2006) e Cosenz e Noto (20I6) relacionam tais dificuldades com as próprias opções epistemológicas adotadas pelas escolas de 
pensamento que dominaram esse período. Para tais autores, os modelos construídos a partir da fragmentação de conceitos, tradicionalmente usados na área de gestão, não poderiam contemplar as relações necessárias para a completa descrição da essência dos fenômenos relacionados à transitoriedade do ambiente, tão natural ao processo estratégico. A dominância de tais abordagens permanece, então, limitando o completo entendimento das relações que constituem os processos de interação socioeconômica, o que leva as organizações a erguerem as fundações de suas decisões sobre terrenos cársticos ${ }^{3}$.

Nesse contexto, a ciência da complexidade é a única que possui a carga conceitual para romper com a fragmentação da área de gestão e dar nova vida à estratégia, permitindo a compreensão das interações entre as organizações, o ambiente e as estratégias emergentes e deliberadas (Stacey, 1995). Diante das dificuldades apresentadas até então, “[...] a complexidade, a dinâmica e a abertura teriam de tornarse as palavras-chave do novo paradigma da ciência das organizações" (Haselhoff, I98I, p. 3I). Entretanto, Caldwell (I994) ressalta que uma mera revisão metodológica é insuficiente para possibilitar um espaço de inovação na ciência. Uma vez que o método possui um caráter de cunho prescritivo (Caldwell, I994), fica evidente que é preciso transcender os limites impostos por ele, para que este não atue como um fator limitante ao questionamento, mas sim um instrumento para produção da sua resposta.

Isso fica claro nos trabalhos de Jarzabkowski e Paul Spee (2009) e Hurtado (2006) que, ao investigarem aquilo que já foi feito na área de gestão, em termos de estudos acerca da complexidade, identificaram que tais textos ainda se encontram restritos por uma perspectiva cartesiana de mundo. Buchanan e Denyer (2013) concordam com tal afirmação e ainda ressaltam que há uma ampla aceitação de que os estudos que levam à uma melhor compreensão da dinâmica nesse tipo de sistema se encontram fora do mainstream dos estudos organizacionais.

Contudo, pela necessidade de aceitação dos pares e adequação ao status quo, os pesquisadores têm se visto forçados a orientar suas pesquisas pelo método e não pelo problema estudado, fazendo com que o favoritismo por uma dada abordagem se

3 Os solos cársticos são solos calcários que, devido à infiltração de água e à presença de áreas cavernosas, podem estar sujeitos a abatimentos bruscos sem sinais prévios denunciadores.

\section{Revista Administração em Diálogo}

ISSN 2178-0080 
sobressaia em relação ao próprio objetivo de pesquisa (Terra, Ganzert, Medeiros, \& Passador, 20I3). Prahalad e Hamel (1994), Faria (2005) e Hurtado (2006) vão além e ainda defendem que a construção de um conjunto de princípios consistentes e devidamente articulados, acerca do processo estratégico, requer a reestruturação da própria postura da administração como ciência. Tal situação requer uma profunda reflexão na área, com o objetivo de prover os pesquisadores de subsídios que lhes permitam reconhecer as limitações metodológicas às quais se encontram submetidos e buscar por alternativas que lhes permitam compreender a essência dos fenômenos que buscam conhecer.

Tendo por base o exposto, o presente artigo tem por objetivo apontar orientações epistemológicas sobre como as investigações acerca do processo estratégico devem ser tratadas e as consequências metodológicas dessa mudança de percepção, visando possibilitar o surgimento de um espaço teórico reflexivo, capaz de responder à complexidade dos cenários contemporâneos. Cabe ressaltar que o que se propõem aqui não é negar os avanços expressivos ocorridos nas últimas décadas de estudos na área, mas reforçar uma importante lacuna epistemológica que vem levando muitos desses avanços a se basearem em princípios insuficientes para abarcar características fundamentais dos fenômenos estudados.

Conforme lembra Thiollent (1983), estudos com tais características se mostram relevantes, uma vez que as reflexões de ordem epistemológica devem ser contempladas por todo e qualquer pesquisador e não podem ser negligenciadas, sob pena de levar a um desligamento entre a teoria e a prática cotidiana. Portanto, um estudo que traga orientações sobre a base epistêmica necessária para compreender os problemas ligados aos fenômenos complexos aos quais as organizações se encontram submetidas se justifica diante da crescente complexidade dos cenários, cuja dinâmica tem levado a transformações que implicam em elevados impactos políticos, ambientais, econômicos e sociais.

\section{A Estratégia e o Problema Estratégico sob a Ótica da Complexidade}

Foi diante do paradigma cartesiano e com forte influência do pensamento positivista que Frederick Taylor deu início aos seus experimentos que culminaram com 
aquilo que foi denominado de administração científica (Bateman \& Snell, ı998, 20o6; Caravantes, Panno, \& Kloeckner, 2005; Miller, I987). Taylor levou a concepção do homem máquina à administração e transportou ideias como aquelas ligadas ao rendimento da termodinâmica de Carnot, para as questões relacionadas ao rendimento do ser humano médio (Bauer, I999).

Como era de se esperar, na estratégia os caminhos foram similares.

Da mesma maneira que Frederick Taylor havia transformado uma montagem complexa em algo que poderia ser repetido, decompondo-a em tarefas simples, a nova doutrina [da estratégia], o equivalente gerencial do Taylorismo, visava fazer da gerência de empresas complexas algo sistemático e previsível. Uma vez definida a estratégia, as estruturas e sistemas baniriam as idiossincrasias humanas problemáticas, permitindo, assim, que as empresas de grande porte, e diversificadas, fossem administradas como uma máquina. (Ghoshal, Bartlett, \& Moran, 20O2, p. 2I).

Entretanto, Zollo, Cennamo e Neumann (20I3) argumentam que o desenvolvimento sustentável de uma organização contemporânea exige que estas transcendam a clássica abordagem baseada na otimização de recursos e na geração de benefícios para os acionistas e busquem possibilitar a geração de valor para uma ampla gama de intervenientes.

Percepção similar é compartilhada por Faria (2005, p. 63) que afirma que os pesquisadores da área "devem transcender disputas epistemológicas e reconhecer elementos causais tidos como ‘não empíricos' ou 'invisíveis”, incompatíveis com os paradigmas de origem positivista/cartesiana que dominam a ciência da administração, desde seus primórdios. Buchanan e Denyer (2013) concordam com tal perspectiva e defendem que a fragmentação oriunda dessas abordagens é um dos principais desafios para a pesquisa de fenômenos como as complexas crises contemporâneas. Para os autores, a busca por metodologias menos convencionais se torna um campo não só promissor, como necessário para lidar com os problemas contemporâneos.

Apesar dos avanços denunciadores que se desenvolveram nas ciências naturais durante o século $\mathrm{XX}$, o rompimento da ciência das organizações com as tratativas baseadas em um universo mecânico, previsível e baseado na otimização, só ganhou força a partir da década de I980, quando as ações de grandes líderes mundiais promoveram uma ruptura na ordem política, social e econômica estabelecida após a segunda guerra mundial (Drucker, 2003). De acordo com Peters (I989), o aumento do 
número de variáveis, decorrentes da globalização, do novo modelo especulativo dos mercados financeiros, das novas tecnologias e da própria visão da sociedade, fizeram com que a previsibilidade se tornasse muito difícil nesse período, evidenciando as deficiências dos modelos adotados até então.

Nesse período, diversos pesquisadores se voltaram à teoria dos sistemas e seus derivados para descrever os fenômenos sociais, dentre eles aqueles ligados ao problema estratégico (Capra, I983; Mintzberg et al., I998; Mintzberg et al., 2003). Modelos objetivos, como o de Farjoun (2002) e Smets e Jarzabkowski (2OI3), e descrições como as de Stacey (1995) e Bin, Salles-Filho (2012), se tornaram frequentes no mundo da estratégia e trouxeram novas perspectivas baseadas no caos, na concepção da complexidade como um fenômeno humano e na teoria da evolução (Caravantes et al., 2005; Mintzberg et al., I998; Mintzberg et al., 2003).

Contudo, Hurtado (2006) ressalta que a área da estratégia ainda é tipicamente reducionista. Observa-se que mesmo as tratativas contemporâneas de gestão que reconhecem a complexidade como algo inerente à natureza da sociedade ainda carregam elementos típicos dos paradigmas de origem cartesiana, o que diminui sua eficácia na compreensão sistêmica da realidade organizacional (Drucker, 2003; Hurtado, 2006). As obras de Bin, Salles-Filho (20I2) e Smets e Jarzabkowski (2OI3) são um bom exemplo dessas dificuldades. Ambos reconhecem a complexidade e buscam construir uma visão sistêmica dos problemas que se propõem a estudar. Mas apesar dos resultados inegavelmente bem estruturados, estes ainda se vêm presos à clássica abordagem de estudos de casos que rompem com a perspectiva de interligação dos fenômenos internos e externos. Mesmo em esforços baseados em um interessante ponto de vista multidisciplinar de base evolucionária, como na obra de Bin, Salles-Filho (2OI2) a fragmentação chega a ser visível até mesmo na divisão da estratégia em blocos básicos de análise que direcionam a atenção às partes do problema, em detrimento das relações, promovendo uma perda de capacidade explicativa acerca daquelas dinâmicas que são causadas pela relação entre as partes e não pelas partes, tomadas individualmente.

Para ilustrar como tal problemática vem afetando a percepção dos pesquisadores da área acerca do processo estratégico, Mintzberg, Ahlstrand e Lampel 
(1998) recuperam o poema de John Godfrey Saxe que conta a história de cegos que tentam descrever um elefante. Para tal, os autores colocam o meio científico no papel dos cegos e o processo estratégico como um grande elefante, ainda por descobrir. Os autores concluem que "como ninguém teve a visão para enxergar a fera toda, cada um tocou alguma parte e 'seguiu em total ignorância' a respeito das demais” (Mintzberg et al., I998, p. 3). É evidente que isso não tira os méritos de nenhum dos esforços realizados pelos pesquisadores da área até então, mas é preciso perceber que essa fragmentação, observada mesmo nas tratativas contemporâneas, consiste em um problema real dos estudos da área (Mintzberg \& Lampel, ı999; Mukherji \& Hurtado, 2OOI).

Conforme lembra Mintzberg (2003), essa fragmentação começa já nas definições do termo que, segundo o autor, estariam concentrada sobre dois eixos principais: o da governança, baseado na dicotomia entre aprendizado e controle e; o do foco, cuja dicotomia envolve os aspectos internos e externos à organização. Segundo Mintzberg e Lampel (I999), isso impactou na própria construção do conhecimento da área. Hoje, o pensamento estratégico encontra-se dividido em dez grandes escolas de pensamento, dificultando a devida compreensão do problema e de suas complexas relações. Essa realidade tem imposto aos pesquisadores da área uma pressão para encontrar novas formas de lidar com as mudanças estruturais às quais ela vem sendo submetida (Prahalad \& Hamel, I994) e a buscarem a construção de uma matriz conceitual mais rica do que a adotada até então (MacKay \& Tambeau, 2OI3).

Mintzberg, Ahlstrand e Lampel (1998) ainda vão além e argumentam que essa realidade, vivenciada pela área, requer uma revisão do próprio problema estratégico que seja capaz de transcender a "costura" das principais escolas de pensamento, estabelecidas até então. Isso porque, esses eixos comuns exigem uma concepção onde a estratégia seja vista não como um ato puramente deliberado, mas como "um processo organizacional, de diversas formas inseparável da estrutura, do comportamento e cultura da empresa em que ocorre" (Andrews, 2003, p. 73) Nesse sentido, Mintzberg (I987) propõe unificar tais proposições por meio de uma definição que o autor chamou de estratégia como padrão. Nessa definição, a estratégia é vista como aquilo que é 
realizado pela organização ao longo do tempo e é formada pela conjunção da estratégia emergente, com aquilo que é realizado da estratégia planejada - ver Figura I.

Figura I - Dinâmica que origina as estratégias realizadas pela organização.

Fonte: Mintzberg (2003, p. I4, tradução nossa).

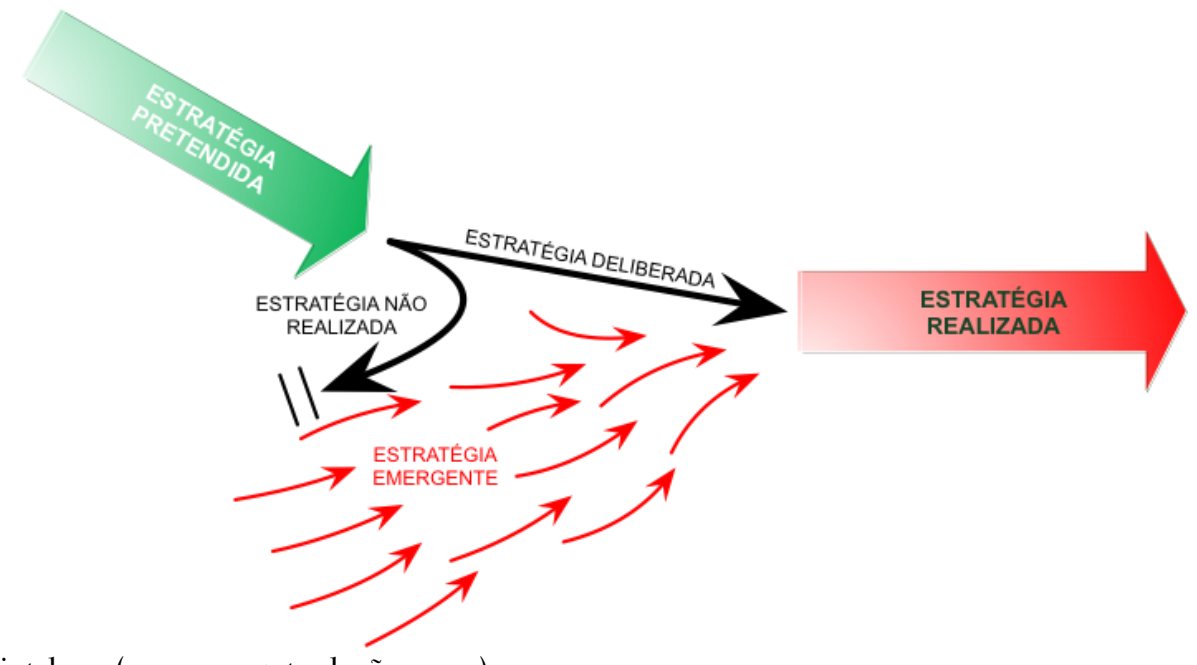

Entretanto, é evidente que essa ainda é uma percepção focada em traçar uma "costura" entre fragmentos do problema estudados previamente por outros autores da área. "Sendo a organização uma totalidade na qual se manifestam fenômenos de tomada de consciência, afetividade e criatividade, torna-se muito difícil e até equivocado o isolamento de variáveis” (Thiollent, I983, p. 8I-82). Isso porque entidades constituídas por meio da interação entre agentes produzem dinâmicas diferentes daquelas obtidas por meio do estudo de seus componentes separados, e a totalidade pode representar mais ou menos do que a "costura" das partes que a constituem (Bertalanffy, 2009).

Tendo por base a percepção de que complexidade requer uma visão integrada da realidade, Morin $(2007$ b) propõe uma definição do termo estratégia onde ela consiste em toda a ação empreendida por uma determinada forma de organização. Essa visão, também, consiste em uma clara oposição à clássica abordagem que trata a estratégia como um plano metodicamente programado, visto que um "[...] programa não obriga a estar vigilante. Ele não obriga a inovar” (Morin, 20o7b, p. 8I). 
Um programa não passa de uma sequência de ações predeterminadas, ou seja, só pode ser bem-sucedido em ambientes com muita ordem e pouca desordem. Já uma estratégia é produzida pelo exame simultâneo das condições determinadas (ordem) e incertezas (desordem), e cria a ação. (Bauer, r999, p. 54).

Diante da concepção de estratégia como ação, a visão linear de mundo, adotada pela perspectiva clássica, acaba por ser insuficiente para lidar com o problema da governança em ambientes instáveis e ricamente interligados. Em condições estáveis, os planos propostos por meio do ideal clássico podem ser suficientes para dar conta das anomalias, mas em condições incertas, somente a estratégia vista como ação pode garantir a perpetuidade das organizações (Morin, 2007b). Portanto, para lidar com a complexidade, os autores da área defendem que a estratégia precisa envolver realização; deve abranger controle e emergência; pode olhar tanto para dentro, como para fora da organização; pode se configurar como truque ou qualquer outro ato ou processo já descritos pela área.

Nesse sentido, desponta a percepção de que a visão de estratégia como ação também só pode ser reconhecida pelo que é realizado, ou seja, só pode ser identificada a partir de sua execução, assim como ocorre na estratégia como padrão. Nesse contexto, a afirmação de Hamel (1998), a respeito da incapacidade dos estudiosos de reconhecer uma estratégia antes que a vejam, se mostra uma realidade insuperável. Diante dessa definição em que a estratégia passa a ser entendida como o processo de formação da ação organizacional, a ciência da complexidade também emerge como o único paradigma com potencial para descrever as dinâmicas às quais as organizações se encontram submetidas. Isso dá uma nova vida à estratégia, uma vez que, a noção de estratégia como ação consegue abranger em uma única definição todos os estudos da área e ir além.

\section{A pesquisa em Estratégia e a Complexidade}

O tratamento das estruturas formadas por uma rede de entidades complexamente interligadas ganhou força a partir dos estudos do biólogo Ludwig Von Bertalanffy, na década de ז930, quando este estabeleceu os princípios do pensamento processual. Para lidar com tal problemática, Bertalanffy propõe que a ciência deve se preocupar com o movimento transformador e auto-organizante das relações complexas

\section{Revista Administração em Diálogo} ISSN 2178-0080 
às quais os componentes dos sistemas se encontram submetidos (Bertalanffy, 2009; Capra, 20o6; Casanova, 2006). Essa concepção se tornou o alicerce daquela que ficou conhecida como teoria dos sistemas (Bunge, I987; Capra, I983, 2006).

A teoria geral dos sistema deve grande parte de seu rigor científico aos estudos de uma área denominada de cibernética (Bauer, r999; Bertalanffy, 2009; Morin, 2ooł̧b; Pierce, 1980). Dentre outras variáveis importantes, a cibernética permite o estudo formal dos laços de realimentação - feedback - que descrevem a circularidade de ação entre as partes de um sistema (Ashby, I970).

Matematicamente, um laço de realimentação corresponde a um tipo especial de processo não-linear conhecido como iteração (palavra que em latim significa "repetição"), na qual uma função opera repetidamente sobre si mesma. Por exemplo, se a função consiste em multiplicar a variável x por 3 - isto é, $\mathrm{f}(\mathrm{x})=3 \mathrm{x}$ - a iteração consiste em multiplicações repetidas. (Capra, 2006, p. IOZ).

Os passos oriundos de uma interação são chamados de mapeamentos e são representados por um conjunto de transições de estado do tipo $\mathrm{x} \rightarrow \mathrm{y}$ (Ashby, 1970). Nesse contexto, um sistema composto por uma ampla rede de relações é capaz de criar laços de feedback que exigem uma modelagem matemática não-linear. A existência de tais laços possui implicações importantes ao passo que rompe com os preceitos de causalidade linear (Bateson, I967), permite a compreensão da dinâmica das entidades autogovernadas e autorreguladas (Bertalanffy, 2009) e também explica porque sistemas submetidos a essa condição podem ser conduzidos à incerteza e ao indeterminismo (Capra, 20o6).

Entretanto, os laços de feedback não são responsáveis apenas pela complexidade e o indeterminismo, eles também são elementos fundamentais para garantir a sustentabilidade dos sistemas complexos, ao passo que lhes proporcionam a “sensibilidade” em relação ao ambiente que lhes permite se adaptar (Vladislav, 2OI7). Dessa forma, o contexto da ciência econômica contemporânea indica que uma investigação mais detalhada dos laços de feedback e dos processos dinâmicos, ligados à dependência temporal, se faz necessária (Pyka \& Grebel, 2006). Visto que uma particularidade fundamental dos sistemas sociais humanos é que estes "[...] são guiados por ciclos de feedback, em que estão presentes a liberdade de escolha e as restrições, e o estado ocupado pelo sistema é o resultado de seus pormenores 
históricos”, os laços de feedback assumem especial importância para a área da estratégia (Stacey, I995, p. 480).

Para Kiviluoto (20I3) e Hurtado (2006), esse contexto faz da compreensão holística da realidade um passo fundamental para o completo entendimento do sucesso empresarial. Para Farmer e Foley (2009), isso torna urgente a adoção de uma abordagem diferenciada que permita a ampla compreensão das interações entre os agentes envolvidos no processo. Cosenz e Noto (2016) argumentam que essa problemática demanda metodologias voltadas ao estudo de dinâmica de sistemas.

Diante dessa realidade, a Soft Systems Methodology (SSM) de Checkland (I989) se mostra promissora para proporcionar uma visão holística acerca das relações que formam um sistema complexo. A SSM, enquanto metodologia sistêmica, trata-se de uma abordagem top-down que tem sido usada com sucesso na área de gestão para a modelagem de atividades em diversos níveis, entre eles: I) os sistemas sociais e culturais; 2) a interação entre indivíduos e os sistemas de informação; 3 ) as atitudes e; 4) as estruturas e processos de um dado sistema (Hanafizadeh \& Mehrabioun, 20I8).

Além da SSM, abordagens bottom-up, como as simulações computacionais, também têm se mostrado hábeis para conseguir explicar a dinâmica desse tipo de problema (Cosenz \& Noto, 20I6). Assim, M. Buchanan (2009) advoga que as simulações com base em modelos multiagentes devem se constituir em uma importante metodologia para descrever o comportamento das dinâmicas socioeconômicas, como aquelas estudadas quando se analisa o problema estratégico. Por meio desse tipo de abordagem, é possível compreender problemas em que não há uma solução analítica, como as interações sociais e outros tipos de modelos não-lineares (Billari, Fent, Prskawetz, \& Scheffran, 20o6; Farmer \& Foley, 2009).

Atualmente as simulações baseadas em sistemas multiagentes já se tratam de uma “[...] área de pesquisa bem reconhecida ao redor da qual orbitam muitas associações e comunidades científicas, conferências e publicações” (Billari et al., 2006; Squazzoni, 2OIO, pp. 197-I98). Nas ciências naturais, autores como Varela, Maturana, e Uribe (I974), Maturana e Varela (I997), Lenton (I998), Margulis (I998) e Watson e Lovelock (1983) se valeram dessas notáveis características dos experimentos virtuais para testarem suas hipóteses a respeito de sistemas situados, tanto em níveis sistêmicos 
acima, como abaixo daqueles a que se refere o processo estratégico nas organizações sociais de produção. Isso reforça a ideia de que esse tipo de abordagem se mostra promissora para lidar com problemas dessa natureza.

Contudo, as abordagens de simulação tratam-se de experimentos virtuais de natureza empírica que carecem de um suporte teórico/conceitual robusto para a formulação de hipóteses a serem testadas pelo experimento. Para lidar com tal problemática, Janssen e Anderies (2013) propõem a elaboração de modelos formais, seguidos de experimentos que podem se dar por meio de simulação computacional ou em campo, para comprovar a validade das hipóteses propostas. A modelagem formal se faz fundamental nesse processo e objetiva propor as características dinâmicas do sistema em estudo para permitir a generalização do que foi observado nos estudos de fins exploratórios, como os estudos de caso, enquanto a etapa experimental permite testar as hipóteses em um nível microssituacional e explorar aspectos e padrões não observados por meio dos primeiros (Janssen \& Anderies, 2OI3).

Contudo, cabe ressaltar que, uma vez que o caminho para as explicações de âmbito cibernético, como aquelas ligadas à complexidade, deve se dar por meio das abordagens de caráter negativo (Bateson, 1967), os estudos de caso, por si só, se mostram limitados para explicar o fenômeno, uma vez que descrevem apenas uma das muitas trajetórias que um sistema poderia seguir (Janssen \& Anderies, 2013).

Koponen (20I4) ainda destaca que não basta pensar nas relações em um contexto de isolamento do problema, é preciso tratar os fenômenos como conceitos complexos, dinâmicos e multifacetados, aliando constructos conceituais e empíricos em múltiplos níveis. Isso demanda múltiplas abordagens metodológicas e epistemológicas para possibilitar um amplo entendimento desse tipo de problema (Zollo et al., 2OI3). Janssen e Anderies (20I3) reforçam tal percepção e destacam a importância das abordagens multimétodos no estudo de sistemas socioecológicos, como aqueles envolvendo o problema estratégico, em especial quando estes visam lidar com problemas descritivos, explicativos e de intervenção. Diante de uma realidade onde os sistemas complexos são a base das dinâmicas estudadas, abordagens que tratam o problema tanto por uma perspectiva top-down, como por uma perspectiva bottom- 
up, se fazem desejáveis, até mesmo para atender aquilo que foi preconizado por Bertalanffy (2009), como atributo fundamental para o estudo de sistemas.

Para se estabelecer hipóteses condizentes com a complexidade dos fenômenos, é preciso reconhecer que "quando os fenômenos do universo são vistos como interligados entre si, por transferência de energia e relações de causa e efeito, a imagem resultante é a de cadeias de causalidade complexamente ramificadas e interconectadas" (Bateson, 1967 , p. 30). Em um contexto complexo, as entidades podem contemplar diversos subsistemas que possuem dinâmicas com propriedades emergentes, referentes ao vínculo entre seus subsistemas, que por sua vez contêm as propriedades oriundas das conexões de seus respectivos subsistemas e assim sucessivamente. Essa propriedade possibilita que fenômenos de diversas áreas do conhecimento pertençam a diferentes níveis sistêmicos, cujos níveis mais complexos compreendam as propriedades de seus subsistemas, adicionadas de particularidades de suas próprias estruturas de acoplamento (Bauer, I999; Bunge, I987; Capra, I983).

Portanto, sob a ótica da complexidade, o conhecimento precisa ser construído a partir de reflexões teóricas, experimentais e empíricas que possibilitem distinguir os níveis de escala e generalização ligados ao fenômeno (Bertalanffy, 20o9; Casanova, 2006). Nesse contexto, Koponen (2014) destaca que fenômenos dessa natureza envolvem dinâmicas multifacetadas que requerem a unificação de constructos conceituais e empíricos em múltiplos níveis. Isso traz, “[...] uma nova vida aos métodos experimentais e à construção de teorias de conjuntos e subconjuntos articulados" (Casanova, 2006, p. 36) que exige uma ruptura com as barreiras disciplinares entre as ciências, impostas pela fragmentação do pensamento cartesiano (Capra, 20o6; Casanova, 2006). Isso porque, a ideia central por trás da teoria dos sistemas é que os sistemas só podem ser entendidos como totalidades integradas, cujas partes ou componentes se relacionam de tal forma que não podem ser reduzidos a unidades menores, sem que percam suas propriedades (Bunge, ı987; Capra, I983, 2006). Portanto, a compreensão dos sistemas complexos só faz sentido se estes forem tratados a partir de sua totalidade, tendo como objeto central de estudo, as relações que se estabelecem entre seus componentes (Ashby, r970; Bertalanffy, 2009). 
Contudo, cabe ressaltar que a ideia central do ponto de vista da complexidade não consiste na negação do que foi produzido pelas disciplinas, "se todas as coisas são causadas e causantes, ajudadas e ajudantes, mediatas e imediatas e mantidas por uma ligação material e insensível que as sujeitam, torna-se impossível conceber as partes sem conceber o todo e tampouco o todo sem conceber as partes" (Morin, 2007a, p. 22). Sob tal concepção, a construção do conhecimento deve se valer de elementos da reflexão teórica, experimental e empírica, em estágios que vão do plano dos fragmentos ao plano da totalidade, com o objetivo de distinguir os níveis de escala e generalização ligados ao fenômeno (Bertalanffy, 20o9; Casanova, 20o6). Nesse contexto, "um novo caminho torn[a]-se particularmente rico: a busca de 'interfaces"” (Casanova, 20o6, p. 3I). Para Nowotny (2005), diante da complexidade, a interface entre as ciências naturais e as ciências humanas tem um papel estratégico na compreensão das interações sistêmicas ocorridas na sociedade e sua relação com a natureza.

Tendo por base essa importância das relações enquanto objeto de estudo e das interfaces entre os mais diversos níveis sistêmicos para compreensão de problemas complexos, Terra e Passador (20I6) propuseram uma visão sobre o problema estratégico onde ele precisaria ser analisado sob a ótica de I2 variáveis que vão das características dos componentes dos sistemas envolvidos, até as propriedades dos acoplamentos: internos do sociossistema da organização; internos do tecnossistema da organização; internos do sistema socioeconômico; internos do ecossistema; entre os sociossistemas e os tecnossistemas da organização; entre os sociossistemas da organização e o sistema socioeconômico; entre os tecnossistemas da organização e o sistema socioeconômico; entre os sociossistemas da organização e o ecossistema; entre os tecnossistemas da organização e o ecossistema; entre o ecossistema e o sistema socioeconômico e; entre o ecossistema e o restante do universo.

Nas ciências humanas, áreas de vanguarda, como aquela que ficou conhecida como "a grande história", têm adotado uma perspectiva multidisciplinar, também pautada nas relações que estudam a própria história em seu contexto, integrando cosmologia, geologia, a vida e a própria humanidade em uma única perspectiva (Christian, 2004; Rodrigue, 20I0; Spier, 2008). Sua natureza sistêmica, busca construir modelos baseados em princípios comuns, independentemente de sua 
natureza (Ažderska \& Jerman-Blažič, 20I3). O ponto revolucionário da "grande história" é que ela adota o universo como ponto de referência sob uma perspectiva efetivamente científica, assim como propuseram Terra e Passador (20I6) para a área de estratégia. O sucesso foi tão evidente que a "grande história" tem sido incorporada nos currículos de importantes escolas ao redor do globo (Rodrigue, 20Iо).

Essa nova visão de mundo, que trata as relações como objeto de estudo, abre espaço não só para o estudo da totalidade integrada, como para os métodos explicativos de natureza analógica, que permitem transpor princípios organizacionais para diferentes níveis sistêmicos.

É evidente que os seres humanos, ao contrário das moléculas, são capazes de imaginar, inventar, criar correlações, ou seja, são capazes de se propor os problemas que vivem. Não se pode sequer conceber, portanto, a ideia de uma redução do fenômeno humano a parâmetros físico-químicos; ao contrário, tratase de perceber que as noções de acontecimento e de sua consequência (a evolução) são noções comuns a todas as ciências, naturais ou sociais, que se deparam com a questão das populações, ou seja, do ser e estar juntos. Assim, o que desponta é a perspectiva de uma articulação entre ciências distintas na medida em que estas se defrontam com problemas de natureza comum. (Bauer, г999, p. г46).

Desse modo, pode-se dizer que a complexidade “[...] não se dedica a descobrir analogias fenomênicas, mas a encontrar os princípios organizacionais comuns, os princípios de evolução destes princípios, os caracteres de sua diversificação" (Morin, 2007b, p. 28). Nowotny (2005, p. 29) ainda destaca que esse entendimento teórico da complexidade, estabelecido a partir da interface entre múltiplas disciplinas, deve ocorrer “[...] não como um fenômeno matemático e sim como um fenômeno social”.

Diante dessa realidade, a abordagem fenomenológica de Terra e Passador (20I5) se mostra promissora enquanto instrumento metodológico para construção de modelos formais. Essa perspectiva se trata de uma metodologia híbrida, formada por uma etapa de redução fenomenológica e uma etapa de caráter analógico. Na primeira, o pesquisador busca uma síntese holística do fenômeno estudado, enquanto, na segunda, o pesquisador visa identificar elementos comuns com outras áreas de estudo que permitam explicar o fenômeno em pauta a partir de uma heurística focada em um conjunto de interações entre essas propriedades. Trata-se, portanto, de uma abordagem top-down capaz de produzir sínteses fenomênicas que podem ser testadas 
a partir de uma perspectiva bottom-up, fechando o ciclo proposto por Bertalanffy (2009) para apreensão do conhecimento sobre sistemas.

Conforme pode ser visto em Mintzberg e Lampel (I999), as metodologias analógicas já vêm sendo utilizadas com sucesso na área de gestão há algumas décadas e ainda são encontradas em uma série de trabalhos recentes, como o de De Florio, Bakhouya, Coronato, e Di Marzo (20I3). Os autores adotam uma abordagem analógica baseada na complexidade para propor uma estrutura organizacional fractal bioinspirada que em muito faz lembrar as abordagens de Beer (I995). Entretanto, as analogias, por si só, não são capazes de abarcar todo o processo de descrição e explicação do fenômeno. Conforme visto anteriormente, o estudo científico da complexidade exige que as hipóteses teóricas, formuladas por meio de metodologias desse tipo, se reflitam em experimentos ou pesquisas de campo que as validem e que respeitem os princípios fundamentais da complexidade.

Além disso, é preciso destacar que o estudo das interações em sistemas complexos também implica em um problema de natureza epistemológica. Qualquer tentativa de se conhecer a dinâmica de um sistema acoplado ao restante do universo exige, ao mesmo tempo, o conhecimento desse sistema e do universo por inteiro para que nenhuma relação escape ao observador. "Com o aumento da complexidade das situações, o número de fatos a serem explicados cresce e estas só podem ser explicadas com modelos mais avançados e elaborados [...]” (Koponen, 2OI4, p. 28). Nessas condições, a certeza exige, no mínimo, os conhecimentos e os poderes de cálculo ilimitados do intelecto superior proposto por Laplace (I829). Contudo, os limites para o processamento e transmissão de dados, impostos pela teoria da informação (Pierce, I980; Shannon, I948), mostram que, mesmo diante de uma abordagem determinista, a possibilidade de existência de tal ser é, no mínimo, pouco realista.

Assim, a ruptura com determinismo se faz fundamental para unificar a criatividade humana com a natureza (Prigogine, r996). Mas cabe ressaltar que “[...] a complexidade não se reduz à incerteza, é a incerteza no seio de sistemas ricamente organizados" (Morin, 2007b, p. 35). "O pensamento complexo não recusa de modo algum a clareza, a ordem, o determinismo. Ele os considera insuficientes, sabe que não se pode programar a descoberta, o conhecimento nem a ação" (Morin, 2007b, p. 83). 
Nesse cenário, “[...] o desafio da complexidade reside no duplo desafio da religação e da incerteza. [...] É preciso aprender a fazer com que as certezas interajam com a incerteza” (Morin, 2007a, p. 63).

\section{Considerações Finais}

Conforme visto anteriormente, o presente esforço de pesquisa teve como objetivo apontar orientações sobre como as investigações acerca do processo estratégico devem ser tratadas para possibilitar o surgimento de um espaço teórico reflexivo capaz de responder à complexidade dos cenários contemporâneos. Diante dessa problemática, uma série princípios fundamentais do pensamento complexo foram listados para abarcar aqueles que permitiriam aos estudos da área explicar os fenômenos organizacionais de maneira mais próxima àquela exigida pelo atual contexto.

Observou-se nesse processo que as abordagens clássicas, baseadas no pensamento cartesiano, são deterministas e buscam analisar detalhadamente as propriedades das partes que compõem um determinado fenômeno. Tal perspectiva apresenta uma série de dificuldades, quando usada para descrever e explicar sistemas ricamente interligados, pois os estudos dos entes individuais nem sempre oferecem as informações necessárias para a compreensão da dinâmica do sistema tecido em conjunto. Mesmo que um ente isolado de um sistema seja capaz de estabelecer relações de um determinado tipo, isso não implica que este as estabeleça dentro da totalidade onde está inserido, o que pode afetar profundamente a compreensão dos princípios organizacionais que regem o fenômeno estudado. Além disso, as propriedades de um todo complexo podem se perder, já que a ausência de um componente desconhecido do problema, ou a negligência de qualquer das relações que o constituem, pode levar a explicações e descrições pouco aderentes à realidade que se pretende descrever ou explicar.

Em um contexto como o problema estratégico, que abarca fatores complexamente ramificados e interligados, as abordagens como as adotadas pela teoria geral dos sistemas se destacam. Tais perspectivas diferem do pensamento cartesiano ao passo que não procuram uma descrição ontológica profunda dos entes isolados, mas 
permitem que sistemas intricados tenham sua organização compreendida por meio de sua totalidade, mesmo que partes do sistema ainda sejam desconhecidas ou não tenham sido completamente descritas. Isso porque, seu enfoque reside nas propriedades que permitem o acoplamento das partes e os resultados emergentes desses acoplamentos e não no detalhamento a respeito do funcionamento de cada componente do sistema, tomado separadamente.

$\mathrm{Na}$ área da estratégia, a necessidade de abordagens de caráter holístico já é amplamente aceita e, apesar de ainda não serem adotadas na escala necessária para transformar os instrumentos desenvolvidos pela área, se constituem em uma necessidade tratada por uma série de autores que já reconhecem que os fenômenos complexos não podem ser tratados de maneira isolada e carregam certo nível de incerteza que não permite a modelagem precisa de seus estados futuros. Entretanto, essa percepção leva a uma necessidade de rompimento com a ideia de que a estratégia pode ser metodicamente programada. Para abordar o problema sob a ótica da complexidade, é preciso reconhecer as incertezas e explorar atributos organizacionais que permitam à estratégia ser vista como ação e relacionar a ordem e a desordem a ela associadas.

Contudo, nota-se na área que esse reconhecimento ainda não se converteu em procedimentos metodológicos aderentes a essa nova realidade. As ciências baseadas na complexidade se pautam nas abordagens de caráter negativo e no rompimento com o ideal de disciplinas, já que o contexto precisa ser levado em consideração. Assim, as abordagens transdisciplinares se mostram um caminho natural para a construção de um paradigma integrativo em estratégia, pois a contextualização do problema se daria em diversos níveis sistêmicos que pertencem a diferentes disciplinas. Isso requer um aprofundamento dos autores da área em temas pouco ou nada explorados pelo campo, além de uma ampliação dos relacionamentos mantidos com as mais diversas áreas do conhecimento. Perspectiva que é reforçada pela ideia de que os princípios organizacionais de uma grande diversidade de fenômenos relacionados ao ser e estar juntos são comuns à diversos níveis sistêmicos, o que permite, em última instância, transportar as explicações de tais princípios através de uma série de níveis sistêmicos, por meio do uso de analogias substantivas. 
Por fim, ressalta-se que um dos principais desafios contemporâneos da ciência, por trás da estratégia, consiste em romper com a lógica fragmentadora que permeia a própria estrutura de apreensão do conhecimento. As abordagens multimétodos, estruturadas para abarcar as etapas teóricas, empíricas, indutivas e dedutivas, precisam se tornar parte do cotidiano das pesquisas da área, para permitir uma completa compreensão dos fenômenos estudados. Em uma abordagem cuja lógica tem natureza negativa, a formulação de hipóteses, os experimentos de natureza empírica e a própria análise desses resultados consistem em passos inseparáveis para a construção do conhecimento, tornando inseparáveis as etapas exploratórias, descritivas e explicativas de uma pesquisa, já que as primeiras não podem ser validadas sem a última e esta não pode ser construída sem as primeiras.

Como pôde ser visto no decorrer desta explanação, a crescente complexidade dos cenários traz amplos desafios para a área da estratégia e requer uma guinada na tradicional forma de analisar o problema em pauta. Apesar de a noção de complexidade já ser amplamente aceita, nota-se que princípios importantes dela ainda não são adotados pela área, como: a definição de estratégia como ação; o reconhecimento das relações como objeto de estudo; as abordagens transdisciplinares; os mapeamentos e as abordagens multimétodos e negativas. Métodos e abordagens capazes de levar os estudos da área a um novo patamar qualitativo, efetivamente sinérgico com o pensamento complexo, também têm sido pouco utilizados na área, como: as simulações computacionais; a SSM e; as abordagens fenomenológicas e analógicas.

Com os avanços da ciência da complexidade nas subdisciplinas de gestão, espera-se que a importância da lógica dominante seja reduzida, dando espaço para modelos capazes de contemplar as múltiplas realidades vivenciadas pelas organizações contemporâneas (Woodside, 20I4). Nesse sentido, espera-se que este breve texto tenha contribuído para orientar os autores da área acerca dessas bases imprescindíveis para sustentar as rupturas que se fazem necessárias nesse importante campo do conhecimento. Ressalta-se ainda que, apesar do foco deste esforço de pesquisa ter se dado em uma análise do campo da estratégia, essa abordagem também pode beneficiar outras áreas dos estudos em gestão, que enfrentam diretamente o problema da 
complexidade ou que se relacionam com áreas sujeitas a tal problemática, ampliando, assim, seu potencial de aplicação.

\section{Referências}

Andrews, K. R. (2003). The concept of corporate strategy. In H. Mintzberg, J. Lampel, J. B. Quinn, \& S. Ghoshal (Eds.), The Strategy Process: Concepts, Contexts, Cases (4 ed., pp. $7^{2-}$ 79). Upper Saddle River: Pearson.

Ashby, W. R. (I970). Introdução à cibernética(G. K. Guinsburg, Trans.). São Paulo: Perspectiva.

Ažderska, T., \& Jerman-Blažič, B. (2013). A Holistic Approach for Designing Human-Centric Trust Systems. Systemic Practice and Action Research, 26 (5), 4I7-45O.

Bateman, T. S., \& Snell, S. A. (1998). Administração: Construindo Vantagem Competitiva(C. A. Rimoli, Trans.). São Paulo: Atlas.

Bateman, T. S., \& Snell, S. A. (20o6). Administração: Novo Cenario Competitivo. São Paulo: Atlas.

Bateson, G. (I967). Cybernetic explanation. The American Behavioral Scientist, Io (8), 29-32.

Bauer, R. (ı999). Gestão da Mudança: Caos e Complexidade nas Organizações. São Paulo: Atlas.

Beer, S. (1995). Brain of the Firm: Wiley.

Bertalanffy, L. v. (2009). Teoria Geral dos Sistemas: Fundamentos Desenvolvimento e Aplicações. Petrópolis: Vozes.

Bertero, C. O., Caldas, M. P., \& Wood Jr., T. (I999). Produção Científica em Administração de Empresas: Provocações, Insinuações e Contribuições para um Debate Local. Revista de Administração Contemporânea, 3 (I), I47-I770.

Billari, F. C., Fent, T., Prskawetz, A., \& Scheffran, J. (2006). Agent-based computational modelling: an introduction. In F. C. Billari, T. Fent, A. Prskawetz, \& J. Scheffran (Eds.), Agent-Based Computational Modelling: Applications in Demography, Social, Economic and Environmental Sciences (pp. I-I6). New York: Physica-Verlag.

Bin, A., \& Salles-Filho, S. (2OI2). Science,Technology and Innovation Management: Contributions to a Methodological Framework. Journal of Technology Management \& Innovation, 7 (2), 73-86.

Buchanan, D. A., \& Denyer, D. (20I3). Researching Tomorrow's Crisis: Methodological Innovations and Wider Implications. International Journal of Management Reviews, I5 (2), 205-224.

Buchanan, M. (2009). Could agent-based computer models prevent another financial crisis? Nature, 460 ( $\left.{ }_{25} 56\right), 680-682$.

Bunge, M. (I987). Epistemologia: Curso de Atualização. São Paulo: T. A. Queiroz: Editora da Universidade de São Paulo. 
Caldwell, B. J. (1994). Beyond Positivism: Economic Methodology in the Twentieth Century. London: Routledge.

Capra, F. (1983). The Turning Point: Science, Society, and the Rising Culture. Toronto; New York: Bantam Books.

Capra, F. (2006). A Teia da Vida: Uma Nova Compreensão Científica dos Sistemas Vivos . São Paulo: Cultrix.

Caravantes, G. R., Panno, C. C., \& Kloeckner, M. C. (2005). Administração: Teorias e Processos. São Paulo: Prentice Hall.

Casanova, P. G. (2006). As Novas Ciencias e as Humanidades: Da Academia à Política . São Paulo: Boitempo.

Checkland, P. B. (1989). Soft Systems Methodology. Human Systems Management, 8 (4), 273289 .

Christian, D. (2004). Maps of Time: An Introduction to Big History. Berkeley: University of California Press.

Cosenz, F., \& Noto, G. (20I6). Applying System Dynamics Modelling to Strategic

Management: A Literature Review. Systems Research and Behavioral Science, 33 (6), 703-74I.

De Florio, V., Bakhouya, M., Coronato, A., \& Di Marzo, G. (2013). Models and Concepts for Socio-Technical Complex Systems: Towards Fractal Social Organizations. Systems Research and Behavioral Science, 30 (6), $750-77^{2}$.

Drucker, P. F. (1994). The age of Social Transformation. The Atlantic Monthly, 274 (5), 53-80.

Drucker, P. F. (2003). The New Realities. New Brunswick: Transaction Publishers.

Faria, A. (2005). Realismo crítico em pesquisa estratégica. In M. M. F. Vieira \& D. M. Zouain (Eds.), Pesquisa Qualitativa em Administração (pp. 45-69). Rio de Janeiro: Editora FGV.

Farjoun, M. (2002). Towards an organic perspective on strategy. Strategic Management Journal, 23 (7), 56I-594.

Farmer, D., \& Foley, D. (2009). The economy needs agent-based modelling. Nature, 460 $(7256), 685^{-686 .}$

Ghoshal, S., Bartlett, C. A., \& Moran, P. (2002). Um novo manifesto pela gerência (A. B. Rodrigues, Trans.). In M. A. Cusumano \& C. C. Markides (Eds.), Pensamento Estratégico (pp. I7-39). Rio de Janeiro: Campus.

Hamel, G. (1998). Strategy emergence. Executive Excellence, I5 (І2), 3-4.

Hanafizadeh, P., \& Mehrabioun, M. (2018). Application of SSM in tackling problematical situations from academicians' viewpoints. Systemic Practice and Action Research, 3I (2), I79$22 \mathrm{O}$.

Haselhoff, F. (I98I). Novo paradigma para o estudo das metas organizacionais (C. R. V. d. Araújo, Trans.). In H. I. Ansoff, R. P. Declerck, \& R. L. Hayes (Eds.), Do Planejamento Estratégico à Administração Estratégica (pp. 20-40). São Paulo: Atlas.

Herepath, A. (20I4). In the Loop: A Realist Approach to Structure and Agency in the Practice of Strategy. Organization Studies, 35 (6), 857-879. 
Hurtado, P. (2006). Will the real complexity in strategic management please stand up? Competition Forum, 4 (I), I75-I82.

Janssen, M. A., \& Anderies, J. M. (2OI3). A multi-method approach to study robustness of social-ecological systems: the case of small-scale irrigation systems. Journal of Institutional Economics, 9 (04), 427-447.

Jarzabkowski, P., \& Paul Spee, A. (2009). Strategy-as-practice: A review and future directions for the field. International Journal of Management Reviews, II (I), 69-95.

Kiviluoto, N. (20I3). Growth as evidence of firm success: myth or reality? Entrepreneurship and Regional Development, 25 (7-8), 569-586.

Koponen, I. T. (20I4). Systemic View of Learning Scientific Concepts: A Description in Terms of Directed Graph Model. Complexity, I9 (3), 27-37.

Laplace, M. d. (I829). Essai Philosophique sur les Probabilités. Bruxelles: Chez H. Remy.

Lenton, T. M. (1998). Gaia and natural selection. Nature, 394 (30), 439-447.

MacKay, B., \& Tambeau, P. (20I3). A structuration approach to scenario praxis. Technological Forecasting and Social Change, 80 (4), 673-686.

Margulis, L. (1998). Symbiotic Planet: A New Look at Evolution. New York: Basic Books.

Maturana, H. R., \& Varela, F. J. (i997). De Máquinas e Seres Vivos: Autopoiese - A

Organização do Vivo.Porto Alegre: Artmed.

Miller, R. W. (1987). Fact and Method: Explanation, Confirmation and Reality in the Natural and the Social. New Jersey: Princeton University Press.

Mintzberg, H. (1987). Crafting Strategy. Harvard Business Review, 65 (4), 66-75.

Mintzberg, H. (2003). Five Ps for strategy. In H. Mintzberg, J. Lampel, J. B. Quinn, \& S. Ghoshal (Eds.), The Strategy Process: Concepts, Contexts, Cases (4 ed., pp. 3-9). Upper Saddle River: Pearson.

Mintzberg, H., Ahlstrand, B., \& Lampel, J. (1998). Strategy Safari: A Guided Tour Through the Wilds of Strategic Management. New York: The Free Press.

Mintzberg, H., \& Lampel, J. (I999). Reflecting on the strategy process. Sloan Management Review, 40 (3), 2I-30.

Mintzberg, H., Lampel, J., Quinn, J. B., \& Ghoshal, S. (2003). The Strategy Process: Concepts, Contexts, Cases. Upper Saddle River: Pearson.

Morin, E. (2007a). Educação e Complexidade: Os Sete Saberes e Outros Ensaios. São Paulo: Cortez.

Morin, E. (2007b). Introdução ao Pensamento Complexo. Porto Alegre: Sulina.

Mukherji, A., \& Hurtado, P. (2OOI). Interpreting, categorizing and responding to the environment: the role of culture in strategic problem definition. Management Decision, 39 (2), IO5-II2. 
Nowotny, H. (2005). The increase of complexity and its reduction: emergent interfaces between the natural sciences, humanities and social sciences. Theory Culture Society, 22 (5), I5-3I.

Peters, T. (1989). Prosperando no Caos . São Paulo: Harbra.

Pierce, J. R. (1980). An Introduction to Information Theory: Symbols, Signals and Noise. New York: Dover Publications.

Prahalad, C. K., \& Hamel, G. (I994). Strategy as a field of study: why search for a new paradigm? Strategic Management Journal, I5, 5-16.

Prigogine, I. (1996). O Fim das Certezas: Tempo, Caos e as Leis da Natureza. São Paulo: UNESP.

Pyka, A., \& Grebel, T. (2006). Agent-based modelling - a methodology for the analysis of qualitative development. In F. C. Billari, T. Fent, A. Prskawetz, \& J. Scheffran (Eds.), AgentBased Computational Modelling: Applications in Demography, Social, Economic and Environmental Sciences (pp. I7-35). New York: Physica-Verlag.

Rodrigue, B. H. (20ıо). Civilization, Big History, and Human Survival. Thought \& Action, I39-I46.

Senge, P. M., Scharmer, C. O., Jaworski, J., \& Flowers, B. S. (2008). Alternative Future. Leadership Excellence, 25 (2), 3-4.

Shannon, C. E. (1948). A mathematical theory of communication. The Bell System Technical Journal, 27, 379-423, 623-656.

Smets, M., \& Jarzabkowski, P. (20I3). Reconstructing institutional complexity in practice: A relational model of institutional work and complexity. Human Relations, 66 (IO), I279-1309.

Spier, F. (2008). Big history: the emergence of a novel interdisciplinary approach.

Interdisciplinary Science Reviews, 33 (2), I4I-I52.

Squazzoni, F. (2OIO). The impact of agent-based models in the social sciences after I 5 years of incursions. History of Economic Ideas, I8 (2), I97-234.

Stacey, R. D. (1995). The science of complexity - an alternative perspective for strategic change processes. Strategic Management Journal, I6 (6), 477-495.

Taylor, T. G., \& Tainter, J. A. (20I6). The Nexus of Population, Energy, Innovation, and Complexity. American Journal of Economics and Sociology, 75 (4), $\mathrm{IOO}^{-\mathrm{IO}} 43$.

Terra, L. A. A., Ganzert, C. C., Medeiros, M. d. L., \& Passador, J. L. (20I3). Reflexões sobre as práticas de pesquisa em administração Revista Administração em Diálogo, I5 (3), I-I7.

Terra, L. A. A., \& Passador, J. L. (20I5). A Phenomenological Approach to the Study of Social Systems. Systemic Practice and Action Research, 28 (6), 6r3-627.

Terra, L. A. A., \& Passador, J. L. (20I6). Symbiotic Dynamic: The Strategic Problem from the Perspective of Complexity. Systems Research and Behavioral Science, 33 (2), 235-248.

Thiollent, M. (1983). Problemas de metodologia. In A. C. C. Fleury \& N. Vargas (Eds.), Organização do Trabalho: Uma Abordagem Interdisciplinar: Sete Estudos Sobre a Realidade Brasileira (pp. 54-83). São Paulo: Atlas.

\section{Revista Administração em Diálogo} ISSN 2178-0080 
Varela, F. J., Maturana, H. R., \& Uribe, R. (1974). Autopoiesis: the organization of living systems, its characterization and a model. Biosystems, 5 (4), I87-196.

Vladislav, V. (2OI7). Wiener and Luhmann on feedback: from complexity to sustainability. Kybernetes, 46 (3), 386-399.

Watson, A. J., \& Lovelock, J. (I983). Biological Homeostasis of the Global Environment: the Parable of Daisyworld. Tellus B, 35 (4), 284-289.

Woodside, A. G. (2OI4). Embrace $\bullet$ perform $\bullet$ model: Complexity theory, contrarian case analysis, and multiple realities. Journal of Business Research, 67 (г2), 2495-2503.

Zey-Ferrell, M. (I98I). Criticisms of the dominant perspective on organizations. The Sociological Quarterly, 22 (2), I8I-205.

Zollo, M., Cennamo, C., \& Neumann, K. (20I3). Beyond What and Why: Understanding Organizational Evolution Towards Sustainable Enterprise Models. Organization \& Environment, 26 (3), 24I-259. 